Check for updates

Cite this: Phys. Chem. Chem. Phys., 2017, 19, 28232

Received 17th May 2017 Accepted 22nd September 2017

DOI: $10.1039 / c 7 c p 03281 a$

rsc.li/pccp

\section{Influence of temperature gradients on charge transport in asymmetric nanochannels $\dagger$}

\author{
Anne M. Benneker, (D) Hans David Wendt, Rob G. H. Lammertink (D) and \\ Jeffery A. Wood (D) *
}

Charge selective asymmetric nanochannels are used for a variety of applications, such as nanofluidic sensing devices and energy conversion applications. In this paper, we numerically investigate the influence of an applied temperature difference over tapered nanochannels on the resulting charge transport and flow behavior. Using a temperature-dependent formulation of the coupled Poisson-Nernst-Planck and Navier-Stokes equations, various nanochannel geometries are investigated. Temperature has a large influence on the total ion transport, as the diffusivity of ions and viscosity of the solution are strongly affected by temperature. We find that the selectivity of the nanochannels is enhanced with increasing asymmetry ratios, while the total current is reduced at higher asymmetry cases. Most interestingly, we find that applying a temperature gradient along the electric field and along the asymmetry direction of the nanochannel enhances the selectivity of the tapered channels even further, while a temperature gradient countering the electric field reduces the selectivity of the nanochannel. Current rectification is enhanced in asymmetric nanochannels if a temperature gradient is applied, independent of the direction of the temperature difference. However, the degree of rectification is dependent on the direction of the temperature gradient with respect to the channel geometry and the electric field direction. The enhanced selectivity of nanochannels due to applied temperature gradients could result in more efficient operation in energy harvesting or desalination applications, motivating experimental investigations.

\section{Introduction}

Nanochannels with a characteristic dimension on the order of the Debye length or less are charge-selective due to charge exclusion of co-ions based on the wall charge of the channel. In recent years, nanochannels ${ }^{1,2}$ and nanoslits ${ }^{3,4}$ have found great interest in experimental and theoretical studies of charge transport and electrokinetic effects in order to improve understanding of charge-selective interfaces for various applications, such as desalination, purification, mixing and energy harvesting. ${ }^{5-15}$ Many different aspects of ion and fluid transport in nanochannels have been experimentally investigated, amongst which are the effects of $\mathrm{pH},{ }^{16,17}$ temperature, ${ }^{18}$ concentration gradients ${ }^{2}$ and the electric field distribution. ${ }^{19}$

Asymmetric, tapered or conical-shaped nanochannels are interesting for different applications, for use as diodes as mimics of the gating functions of biological ion channels and for microscale energy conversion or nanofluidic sensing devices. ${ }^{20-22}$ Different experimental studies have been conducted on

Soft Matter, Fluidics and Interfaces, Faculty of Science and Technology, MESA+ Institute for Nanotechnology, University of Twente, The Netherlands. E-mail: j.a.wood@utwente.nl

$\dagger$ Electronic supplementary information (ESI) available. See DOI: 10.1039/c7cp03281a geometrically asymmetric nanochannels in which the nanochannel surface characteristics ${ }^{23-25}$ or the environmental conditions such as $\mathrm{pH}^{26}$ and temperature ${ }^{27}$ have been successfully controlled and studied, ${ }^{28,29}$ showing among other effects a rectification of the current. ${ }^{30-32}$ This rectification implies that the direction of the applied potential gradient yields a different overall resistance of the nanochannel. As the rectification ratio (the ratio of the resistances between the different electric field directions) is influenced by the distribution of the surface charges and hydrophobicity gradients inside the nanochannel, a gradient in $\mathrm{pH}$ across the nanochannel can enhance or suppress the rectification degree of the channel. ${ }^{20,22}$ The bulk electrolyte concentration and salt concentration gradients over the nanochannel can enhance rectification if the concentration gradient is in the preferred direction of ion transport (induced by geometry), while it can lead to inversion of the rectification if the concentration gradient is reversed. ${ }^{28}$ The measured currents are increased if the temperature of the surrounding electrolyte is increased (under isothermal conditions), independent of the direction of the electric field. ${ }^{27}$

Theoretical frameworks for the description of electrokinetic transport phenomena in uniform nanochannels range from analytical solutions in $1 \mathrm{D}^{33,34}$ to numerical $2 \mathrm{D}$ and $3 \mathrm{D}$ solutions of the combined Poisson-Nernst-Planck and Navier-Stokes 
equations. ${ }^{35,36}$ Numerical investigation of transport in and near nanofluidic channels has yielded information on, among other things, concentration polarization ${ }^{37}$ and micromixing. ${ }^{38}$ Nanochannel characteristics that are of crucial importance are wall $\zeta$-potential/wall charge and size,${ }^{39,40}$ which can be easily varied in numerical studies when compared to experimental systems. ${ }^{41}$ The scale difference between nanochannels and microchannels yields additional numerical difficulty, as one should account for the entrance and exit effects of the nanochannels. ${ }^{42}$ There is a large variation in hydrodynamic resistance, as well as electric field focusing towards the nanochannels yielding curved electric field-lines in the channels and strong velocity gradients. $^{43}$

Ion current rectification inside tapered nanochannels has been simulated widely, using different formulations of the Poisson-Nernst-Planck equations, ${ }^{44-48}$ indicating that tailor made nanochannels show potential as diodes, which has been confirmed in experiments. ${ }^{26}$ Molecular dynamics studies have shown that the sign of the surface charge of the nanochannel walls has a large influence on the transport of liquid and ions through the channels. ${ }^{49}$ The contribution of electroosmotic flow (EOF) to ion current rectification in tapered channels was found to be significant at high voltages for channels with a high overlap of Debye layers. ${ }^{50}$ As the degree of double layer overlap varies along the length of these tapered nanochannels, the transport of ions through these channels is altered when compared to uniform channels. The electric field normal to the nanochannel wall will have a higher magnitude in the more narrow part of the channel, locally affecting the transport of ions and influencing the selectivity of the channels.

If a temperature gradient is applied over a charge-selective interface in an electrolyte solution, a potential difference arises, ${ }^{51,52}$ which can be harvested as electrical work. Applying temperature gradients to experimental nanochannel systems can be challenging, but can yield information on transport mechanisms in macro-systems such as electrodialysis membrane stacks. We have previously numerically investigated the role of temperature and temperature gradients in uniform, electrically gated, nanochannels, ${ }^{42}$ and found that ion selectivity could be theoretically enhanced in systems which contain a temperature gradient along the nanochannel. This was found to primarily result from changes in diffusivity of the ions and a changing viscosity of the liquid. We also found that Joule heating, Soret effects and the influence of induced electrothermal flows were negligible under the conditions studied since ion concentrations are too low to yield a significant contribution for Joule heating. Soret effects were found to be negligible compared to electrophoretic effects even at the lowest voltages applied at the investigated conditions. For asymmetric nanochannels, in contrast to concentration- and pH-gradients etc., temperature gradients have not been investigated to our knowledge. As temperature gradients are not uncommon in ion transport processes, the influence of these gradients is interesting for different applications. In asymmetric nanochannels, the degree of Debye layer overlap varies with the position in the nanochannel, which will result in altered charge transport phenomena through these channels.

In this paper, we numerically investigate the effect of asymmetry of the gated nanochannel coupled to imposed temperature gradients on charge transport through a nanochannel containing a 1:1 monovalent electrolyte $\mathrm{KCl}$ solution using a non-isothermal formulation of the Poisson-Nernst-Planck with Navier-Stokes equations. We compare charge-selectivity, total flux and the resultant rectification between asymmetric nanochannels with uniform channels with temperature gradients, as well as coupling to the applied electric field direction in these systems.

\section{Model description}

The Debye length, the characteristic size of the electric double layer on a wall in an electrolyte solution, is defined as $\kappa^{-1}=\sqrt{\left(\varepsilon_{\mathrm{r}} \varepsilon_{0} k_{\mathrm{B}} T\right) /\left(\sum e^{2} z_{\mathrm{i}}^{2} n_{\mathrm{i}}\right)}$, where $\varepsilon_{\mathrm{r}} \varepsilon_{0}$ is the dielectric permittivity of the medium, $k_{\mathrm{B}}$ is Boltzmann's constant, $T$ is the temperature in $\mathrm{K}, e$ the elementary charge, $z$ is the ion valency and $n$ is the number of ions per unit of volume. ${ }^{53}$ In nanochannel systems, the degree of overlap of Debye layers is thus dependent on the ion concentration and the characteristic size of the nanochannel. If the height of the nanochannel changes with length, this will have an effect on the local overlap of Debye layers, which may influence the selectivity of the nanochannel and thus influence the degree of separation and charge transport.

For our simulations, we used our previously developed model framework, ${ }^{42}$ consisting of a non-dimensional formulation of the non-isothermal steady state Poisson-Nernst-Planck (PNP) equations coupled to Navier-Stokes (NS) equations. We consider an asymmetric nanoslit geometry, as depicted in Fig. 1, with a computational symmetry plane along $\mathrm{AH}$ and we investigated different ratios between $h_{1}$ and $h_{\mathrm{r}}$, keeping the average channel height (scaled to the Debye length at $T=T_{0}$ and $c=c_{0}$ ) constant $(h=1)$ and varying the asymmetry ratio $\alpha=h_{1} / h_{\mathrm{r}}$. The electric potential is split into two terms; the applied potential $\varphi$ and the induced electrokinetic potential $\psi$, both scaled against $V_{\text {th }}=k_{\mathrm{B}} T / e$, as was previously described. ${ }^{42,54}$ This yields eqn (1-3) for description of the electric field, where $c_{1}$ and $c_{2}$ represent the cation and anion concentration respectively, which are scaled

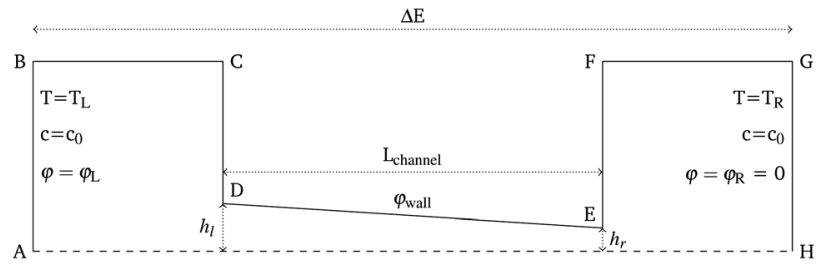

Fig. 1 Schematic illustration of the simulation geometry, which consists of two micro reservoirs connected through an asymmetric nanochannel with a half-height $h_{1}$ at the left side, decreasing to a half-height $h_{\mathrm{r}}$ at the right side. Temperature, potential and concentration in the microchannels are varied in the different cases investigated. In our simulations, $\varphi_{R}=0$ and $\varphi_{\mathrm{L}}=\left[-300 V_{\mathrm{th}}, 300 V_{\mathrm{th}}\right]$. 
against the reservoir bulk concentration $c_{0}, \varepsilon_{\mathrm{r}}$ is the temperaturedependent relative permittivity which is scaled against the permittivity at $T_{0}=293 \mathrm{~K}$ and $L_{\text {ref }}=h$ holds for scaling of the spatial dimensions:

$$
\begin{gathered}
\nabla \cdot\left(\varepsilon_{\mathrm{r}} \nabla \varphi\right)=0 \\
\nabla \cdot\left(\varepsilon_{\mathrm{r}} \nabla \psi\right)=-\frac{1}{2}\left(\kappa L_{\mathrm{ref}}\right)^{2}\left(c_{1}-c_{2}\right) \\
\mathrm{E}=-\nabla(\varphi+\psi)
\end{gathered}
$$

Temperature dependent properties are described by the equations as were used previously, ${ }^{42}$ listed in the ESI. $\dagger$ We consider the case of a 1:1 electrolyte, $\mathrm{KCl}$. This electrolyte was selected because of the similar diffusivities of both $\mathrm{K}^{+}$and $\mathrm{Cl}^{-}$ ions, and the similar responses of diffusivity to temperature of these ions. The use of different, mono- or multivalent ions where diffusivity and temperature dependence is not similar is intriguing for future research. Using the dimensionless NernstPlanck equations we can describe the transport as a result of convection, diffusion and electromigration:

$$
\mathrm{Pe}_{c_{\mathrm{i}}} \mathbf{u} \cdot \nabla c_{\mathrm{i}}-\nabla \cdot\left(D_{\mathrm{i}} \nabla c_{\mathrm{i}}+\frac{z_{\mathrm{i}} D_{\mathrm{i}} c_{\mathrm{i}}}{1+\theta} \nabla(\varphi+\psi)\right)=0
$$

Temperature dependence of ion diffusivity $\left(D_{\mathrm{i}}\right)$ was taken into account, while it was considered independent of concentration in the considered range. The temperature dependence of the ionic heat of transport coefficients was studied, but was found to be negligible. $\theta=\left(T-T_{0}\right) / T_{0}$ is the dimensionless temperature and $\mathrm{Pe}_{c_{\mathrm{i}}}$ is the Péclet number (for mass transfer, both for anion and cation), which is evaluated at the reference temperature $T_{0}$. For description of the fluid hydrodynamics, the dimensionless Navier-Stokes equations with a temperature dependent viscosity $(\mu)$ are applied and a contribution of the Coulomb force $\left(\mathbf{f}_{\mathrm{c}}\right)$ is taken into account:

$$
\begin{aligned}
\operatorname{Re}(\mathbf{u} \cdot \nabla \mathbf{u}) & =-\nabla p+\nabla \cdot(\mu \nabla \mathbf{u})+\mathbf{f}_{\mathrm{c}} \\
\mathbf{f}_{\mathrm{c}} & =\frac{k_{\mathrm{B}} T_{0} c_{0} L_{\mathrm{ref}}}{\mu_{0} u_{0}}\left(c_{1}-c_{2}\right) \mathbf{E} \\
\nabla \cdot \mathbf{u} & =0
\end{aligned}
$$

where Re, the Reynolds number, is evaluated at the reference temperature. The boundary conditions used are identical with our previous work and are provided in the ESI. $\dagger$

While developing the model, we have tested the contributions of Coulomb and dielectric forces on the fluid flow. The dielectric (electrothermal) forces were found to not significantly contribute in this work despite the tapered geometry causing larger field gradients. This also holds for the contribution of Soret diffusion, which was negligible as well. ${ }^{42}$ While the dielectric force was negligible in this case, we note that this may not be the case for other geometries. We have neglected the potentially large effect from temperature-dependent zeta potentials by choosing a constant wall potential, treating the system as an effective gated dielectric in line with previous work, ${ }^{40,42,55}$ due to the large amount of uncertainty in the precise temperature-dependence of solid zeta-potentials. ${ }^{42,56}$
The density of the solvent (water) is considered to be constant with respect to temperature in the considered range between 293 and $313 \mathrm{~K}$, as was mentioned previously. ${ }^{42}$ To close the system of equations, a dimensionless energy balance is formulated (eqn (6)), in which we assumed the heat capacity to be constant in the considered temperature range, while the thermal conductivity $k$ was considered to be dependent on temperature (albeit weakly) and $\mathrm{Pe}_{\theta}$ is the thermal Péclet number. We have also neglected the contributions of Joule heating and viscous dissipation, based on our previous investigations and order of magnitude analysis. ${ }^{42}$

$$
\mathrm{Pe}_{\theta} \mathbf{u} \cdot \nabla \theta=\nabla \cdot(k \nabla \theta)
$$

\subsection{Simulation details}

In this study, we use a 2D model (COMSOL Multiphysics 5.2) to investigate the effects of the non-uniformity of the nanochannels and temperature gradients on charge transport. The general geometry is depicted in Fig. 1 , where we can vary the nanochannel height and asymmetry ratio $\alpha=h_{\mathrm{l}} / h_{\mathrm{r}}$. The electric field and temperature gradient are applied in the adjacent microchannels. For every asymmetry ratio we investigated four different cases; two isothermal cases (at $293 \mathrm{~K}$ and $313 \mathrm{~K}$ ) and two temperature gradient cases in which the direction of the temperature gradient with respect to the nanochannel is altered. In the temperature gradient cases, one reservoir wall is kept at $293 \mathrm{~K}$ while the other is at $313 \mathrm{~K}$, resulting in a difference of $20 \mathrm{~K}$. For all models, $c_{0}=1 \times 10^{-5} \mathrm{M}$, corresponding to a Debye length of $95 \mathrm{~nm}$ at $T=T_{0}$, which was chosen to ensure Debye layer overlap in the $100 \mathrm{~nm}$ channel. The applied potential is varied from -300 to $300 V_{\mathrm{th}}$. The sign of the electric field, as well as the direction of the temperature gradient, is expected to have an influence on the charge transport, as in asymmetric channels we expect current rectification to occur. Three different asymmetry ratios were considered, in which $\alpha$ is 1,3 and 9, while the average channel height was kept constant at $100 \mathrm{~nm}$. The smallest dimension being modeled is thus a channel inlet of $10 \mathrm{~nm}$, which is sufficiently large for the PNP framework to be applicable. ${ }^{57}$ Since the surface charge or $\zeta$-potential of a solid is also dependent on temperature, we investigate an electrically gated nanochannel in which the wall potential is kept constant, to be able to decouple the effect of the temperature gradient over the channel from the effects that temperature has on the surface charge. Preliminary numerical investigations have shown that implementation of a temperature dependent $\zeta$-potential does not yield significantly altered results, although the case for a temperature-dependent surface charge based on a $\mathrm{p} K_{\mathrm{a}}$ model may not show this insensitivity. ${ }^{58}$ The nanochannel is considered to have a constant wall potential of $-50 \mathrm{mV}$, which can be considered as a realistic potential in the case of a gated dielectric. $^{46}$

If we state that the electric field and temperature gradient are aligned, this indicates that both the high $T$ and high $\varphi$ are on the same side of the nanochannel, either left or right. In all our simulations, the largest nanochannel inlet is on the left side of the geometry $\left(h_{1} \geq h_{\mathrm{r}}\right.$, see Fig. 1$)$. This means that if we 
state that the temperature, electric field and geometry are aligned, the high $T$ and high $\varphi$ are also applied at the left side of the nanochannel. If, in our figures, the electric potential is negative, this means that the potential on the left side is grounded and the potential is applied in the right reservoir.

The channel length is 500 dimensionless units, which corresponds to $2.5 \mu \mathrm{m}$ in physical dimensions. The connected microchannels are also 500 dimensionless units in both width and height. We confirmed that this is sufficient to treat the reservoir boundary conditions as far-field, by inspection of the resulting concentration profiles. The nanochannel is mapped using $40 \times 2000$ quadrilateral elements, in which we meshed a symmetric distribution and a maximum element ratio of 10 . The microchannels were meshed using quadrilateral elements, decreasing in size closer to the nanochannel entrance and the boundaries of the system, with mesh independence confirmed for the different geometries and applied potentials.

\section{Results and discussion}

From our simulations, we obtain the flux of both cations and anions and we are therefore able to calculate the total current and the selectivity of the nanochannels. For the four different temperature configurations per geometry we can compare the total current and fluxes as a function of the applied potential. Fig. 2 a shows the total current through the nanochannel as a function of applied potential for the four different temperature configurations in a channel with an asymmetry ratio of 3 . At negative field strengths, the current transport is in the opposite direction and therefore the sign is correspondingly flipped. The total current is higher at a negative field strength (where the anodic microchannel is on the small nanochannel inlet) when compared to the positive field strengths (where the anodic side is on the larger nanochannel inlet). As is expected, the total (absolute) current is highest for the isothermal case at high temperatures, as the diffusivity of both co-ions and counter-ions is enhanced at enhanced temperatures. Likewise, the absolute current is lowest for the isothermal case at low temperatures. The cases in which there are temperature differences show intermediate currents. The case in which the electric field and the temperature gradient are aligned yields the highest current, as the high temperature enhances cation diffusivity. As the nanochannel is cation selective, the enhancement of cation transport towards the channel will result in a higher overall current through the channel, which would be flipped in the case of anion selective materials.

In Fig. $2 \mathrm{~b}$ the influence of temperature gradients on the selectivity of the nanochannel is shown. Selectivity is highest at low field strengths, as at higher field strengths the Donnan exclusion potential in the nanochannel ${ }^{31}$ is overcome by the driving potential and the transport of all ions is enhanced, including the counterions. Depending on the direction of the temperature gradient, the highest selectivity is reached at either slight negative or slight positive field strengths. For the isothermal cases, the low temperature case shows higher selectivity

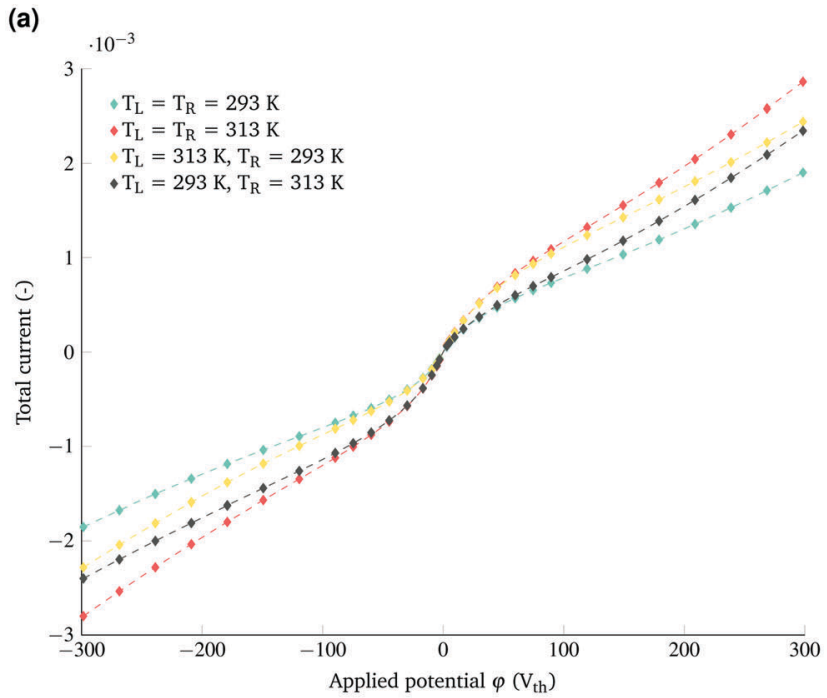

(b)

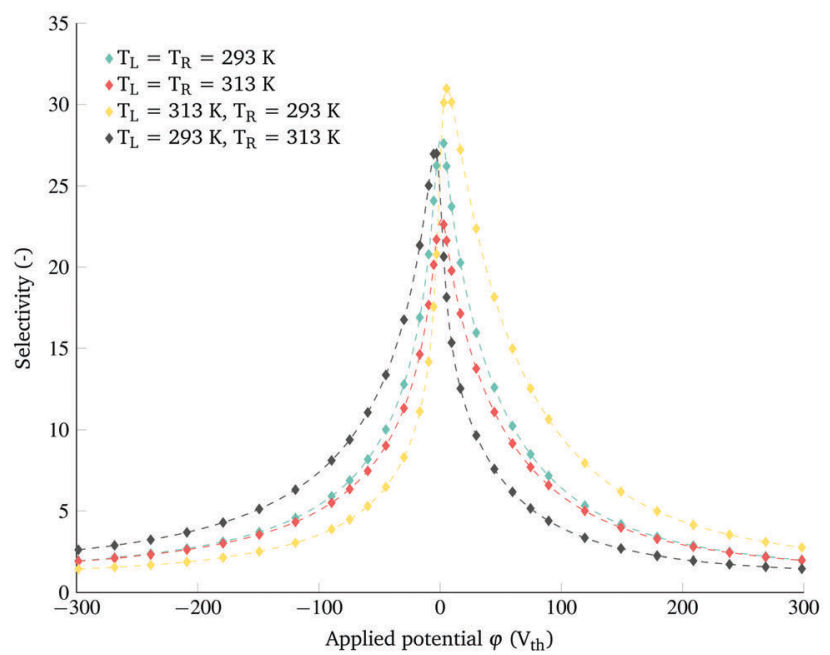

Fig. 2 Total current (a) and selectivity (b) in asymmetric nanochannels with $\alpha=3$ under different temperature configurations. Asymmetry of the current and selectivity with respect to the applied potential indicates current rectification in this system. Dashed lines are for visualization purposes only.

when compared to the higher temperature case, as a result of the lower relative diffusivity of anions (compared to cation diffusivity) at lower temperatures. This is similar to the behavior in uniform nanochannels, where selectivity is the highest for low temperature isothermal cases as well. Selectivity can be enhanced with a temperature gradient, and this enhancement is dependent on the interplay between the direction of the temperature gradient and the direction of the electric field with respect to the nanochannel geometry. The largest enhancement in selectivity is obtained for the case in which the high temperature is on the large inlet of the nanochannel and the temperature gradient is aligned with the electric field, where high $T$, high $\varphi$ and high $h$ are all on the same side of the channel. If the electric field is flipped (corresponding to negative potentials in Fig. 2b), the case with the opposite temperature gradient yields the highest selectivity, again when the temperature gradient is aligned with the electric field (low $T$, low $\varphi$ and high $h$ at the same side). 
This indicates that if the temperature is increased in the reservoir where cations are depleted, the selectivity of the nanochannel is enhanced, independent of the inlet characteristic length of the nanochannel, meaning that the enhanced diffusivity of ions has a large influence on the selectivity of the channel. In particular, the relatively low diffusivity of anions (compared to cations) at lower temperatures enhances the selectivity as this reduces the transport of co-ions through the channel. This was also found to be the case for uniform channels, ${ }^{42}$ albeit with a higher applied wall potential. If the temperature gradient counters the electric field gradient, the selectivity of the nanochannel is reduced, as a result of the enhanced relative diffusivity of anions in the cathodic reservoir, which enhances transport of these anions through the nanochannel.

If we compare the current rectification, which is defined by the ratios of the total current for the different directions of the electric field and shown in Fig. 3, we see that for the isothermal cases, the total rectification is very similar in both quantitative and qualitative behavior and does not exceed a rectification of 1.2 throughout the range of applied potentials. The absolute value of rectification is relatively low as we are working at relatively low ion concentrations in order to have a good selectivity of the nanopore. With increasing field strength the degree of rectification is reduced and at very high field strengths there is no significant rectification observed, just as there was no significant selectivity at these field strengths. For the cases where a temperature gradient is applied, this behavior is altered and the rectification is generally increased. The field strength at which the rectification is the highest is shifted to higher potentials when a temperature gradient is applied. For the case where the high temperature is on the smaller nanochannel inlet this is most pronounced, with rectification factors over 1.5 at low field strengths.

Simulations were also performed for geometries having a different aspect ratio, $\alpha=9$ and $\alpha=1$ (symmetric channels).

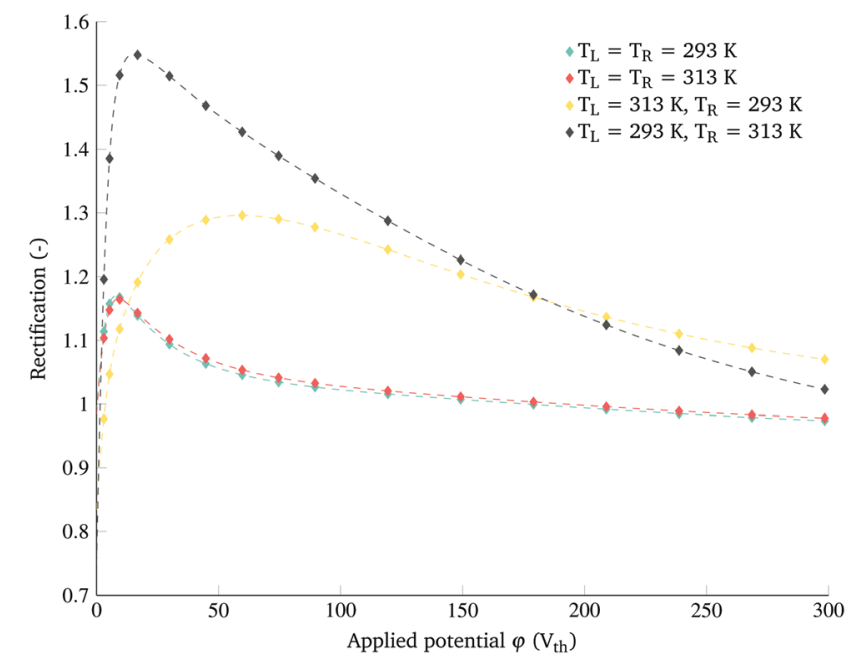

Fig. 3 Current rectification for the four different temperature configurations at different field strengths for the case $\alpha=3$. Rectification is calculated by dividing the total current for the negative applied potential by the total current for the positive applied potential at equal strengths. Lines are for visualization purposes only.
For the symmetric nanochannels, current rectification only occurs in the case of a temperature gradient, due to the symmetry of the system at isothermal conditions, as can be observed in Fig. 4. The current rectification in the case of a temperature gradient is less pronounced in the symmetric nanochannel in comparison to the asymmetric nanochannels for identical salt concentrations (a rectification up to 1.4 at low electric field strengths), which is as expected as the asymmetry enhances the rectification of the current. For the case with a higher asymmetry ratio $\alpha=9$, the rectification is enhanced slightly for all temperature configurations in comparison to the case where $\alpha=3$. This is attributed to the enhanced asymmetry of the nanochannel. The quantitative behavior of the nanochannel with a larger degree of asymmetry is similar to the behavior shown in Fig. 3.

In asymmetric nanochannels, the degree of Debye layer overlap is not constant throughout the channel. In our model, we have defined the Debye layer such that there is an overlap at the location where the channel height is 1 dimensionless unit, which for the asymmetric nanochannels is in the middle of the channel, while in the symmetric channel the Debye layers are overlapping throughout the entire channel. However, as a result of the constricted inlet (or outlet) of the asymmetric nanochannels, selectivity is enhanced when increasing the aspect ratio of the channel. This indicates that the minimum size of the channel (degree of Debye layer overlap) is very important in the determination of nanochannel selectivity. However, many other factors, such as wall potential and $\mathrm{pH}^{20}$ could be of influence and are not investigated here.

A negligible extended space charge develops in the entrance/ exit regions of the nanochannels, even at the highest field strengths. The concentrations of cations and anions are found to be equal outside of the nanochannel. As such, there is no contribution of electro-osmosis of the second kind to the charge transport towards the nanochannel. ${ }^{59}$ The space charge within

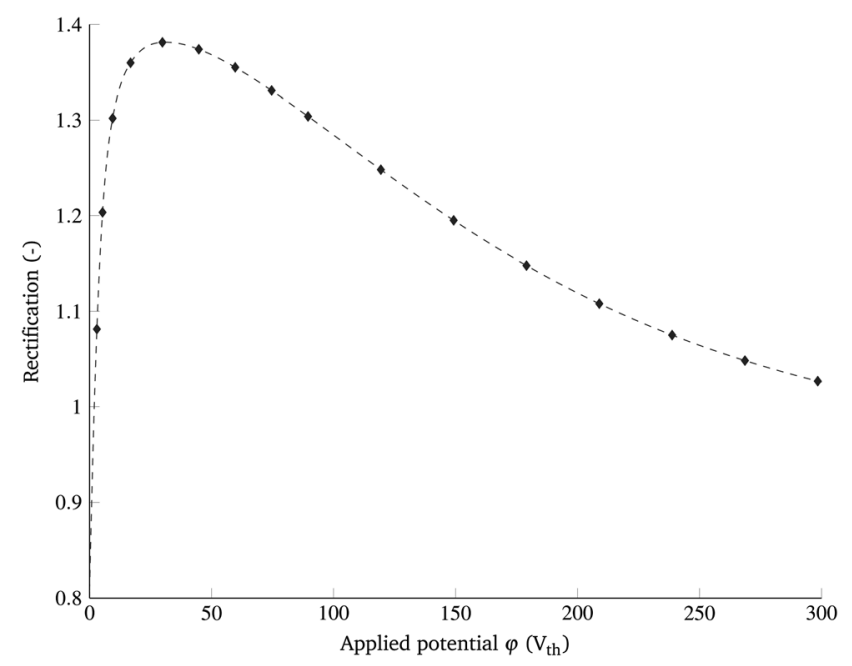

Fig. 4 Current rectification in symmetric nanochannels with an imposed temperature gradient $T_{\mathrm{L}}=313 \mathrm{~K}$ and $T_{\mathrm{R}}=293 \mathrm{~K}$. Rectification is calculated by dividing the total current for the positive applied electric field by the total current for the negative applied electric field at equal strengths. Lines are for visualization purposes only. 
the nanochannel is dependent on the applied electric field, since at high potentials the permeability for anions in the channel increases as the applied field overcomes the Donnan exclusion potential for counter-ions.

By investigating the individual terms of the Nernst-Planck equation (eqn (4)), we are able to determine the contributions of convective, diffusive and electromigration fluxes to the total current. In general, the convective flux has the dominant contribution to the total cation flux, at all modeled field strengths. Convection within the nanochannel is a result of electroosmotic movement of the fluid. For low to moderate applied electric field strengths the convective flux of ions increases with increasing field strength. At sufficiently large applied potentials (above $50 V_{\mathrm{th}}$ ), the relative convective contribution to the total flux decreases again as a result of the lower selectivity of the channel, which results in a smaller cation concentration in the channel. The contribution of thermophoretic transport (Soret effects) is negligible in comparison with the other modes of transport for all field strengths considered except for zero volts. The anion flux as a result of convective ion transport increases with increasing applied potentials. For anions, however, there is no maximum in the convective flux found at moderate field strengths, as the concentration of anions in the nanochannel increases at higher potentials. The electromigration flux of both cations and anions increases with increasing applied potential because this is directly proportional to the electric field strength. For high field strengths, the electromigration flux accounts for a significant contribution in the total current for both cations and anions, as it grows faster than the convective flux. However, at these high field strengths the selectivity of the nanochannel drops significantly and current rectification is reduced as both cations and anions are free to pass through the channel.

\section{Conclusions and perspectives}

In this paper, we have demonstrated that temperature gradients enhance current rectification in charge selective nanochannels through the use of numerical simulations based on a nonisothermal PNP-NS framework. Selectivity of the asymmetric nanochannels is dramatically enhanced if the electric field and temperature gradient are aligned with the nanochannel asymmetry. This enhancement was found to result primarily from increased diffusivity of both cations and anions at elevated temperatures and the subsequent enhancement of transport of ions along the electric field. For asymmetric nanochannels, current rectification occurs also without temperature gradients, but this is significantly enhanced when a temperature difference is applied over the nanochannel. Most interesting is the fact that if the direction of the temperature gradient, the electric field and the decrease in nanochannel height are aligned, the enhancement in selectivity and rectification is over $50 \%$. Channels with higher aspect ratios show a higher ion selectivity and rectification, as a result of a higher degree of Debye layer overlap.
This work was done using $\mathrm{KCl}$, an electrolyte in which both the anion and cation are monovalent and have similar diffusivities and temperature dependence of diffusivity. Extension of this work towards multivalent ions or other ions with different diffusivities and responses of diffusivity to temperature can yield interesting mechanisms for the selective removal of mono- or multivalent ions from salt solutions. Temperature gradients within the Debye layer can be of interest in a fundamental study on how this affects the electro-osmotic flow inside nanoporous materials, as well as the effect of temperature dependence of the $\zeta$-potential of the channel wall, ${ }^{60}$ which we have neglected in this study by assuming a constant wall potential.

For comparison of this work to experimental systems consisting of non-gated nanochannels (e.g. silica nanochannels), the influence of temperature on other properties such as the $\mathrm{pH}$ and dissolved gases and the direct effect on the wall charge should be investigated, since these effects cannot be neglected in experimental systems and we are dealing with a gated dielectric in this research. With increasing temperature, the solubility of gases decreases, yielding bubble formation and possible shifts in $\mathrm{pH}$. Changes in the relative $\mathrm{pH}$ of the two compartments would also result in additional changes in charge transport ${ }^{16,17}$ and should be considered when interpreting experiments.

In general, in ion transport systems such as electrodialysis (ED), thermophoretic transport is not significant when compared to transport as a result of a concentration gradient or a potential gradient. ${ }^{51}$ However, a temperature gradient can yield significant beneficial effects on the ion transport in these systems, if applied alongside an electric potential. This is of interest in many different systems, for instance in enhancing pre-concentration in micro-nano systems and for understanding fundamental ion transport mechanisms. As the selectivity increases, a lower potential would be required to obtain the same degree of desalination in ED systems, which is of interest as it reduces energy requirements in desalination processes.

\section{Conflicts of interest}

There are no conflicts to declare.

\section{Acknowledgements}

This research was financially supported by the European Research Council, under the ERC starting grant 307342-TRAM awarded to R. G. H. Lammertink.

\section{References}

1 S. J. Kim, L. D. Li and J. Han, Langmuir, 2009, 25, 7759-7765.

2 C. Lee, C. Cottin-Bizonne, A. L. Biance, P. Joseph, L. Bocquet and C. Ybert, Phys. Rev. Lett., 2014, 112, 244501.

3 G. Yossifon, P. Mushenheim, Y.-C. Chang and H.-C. Chang, Phys. Rev. E: Stat., Nonlinear, Soft Matter Phys., 2010, 81, 046301.

4 G. Yossifon and H.-C. Chang, Phys. Rev. E: Stat., Nonlinear, Soft Matter Phys., 2010, 81, 066317. 
5 P. Abgrall and N. T. Nguyen, Anal. Chem., 2008, 80, 2326-2341.

6 H.-C. Chang and G. Yossifon, Biomicrofluidics, 2009, 3, 12001.

7 H.-C. Chang, G. Yossifon and E. A. Demekhin, Annu. Rev. Fluid Mech., 2012, 44, 401-426.

8 W. Guan, S. X. Li and M. A. Reed, Nanotechnology, 2014, 25, 122001.

9 J.-Y. Wang, C. Liu, Z. Xu, Y.-K. Li and Y.-L. Liu, Microsyst. Technol., 2013, 20, 35-39.

10 Q. Yu and Z. Silber-Li, Microfluid. Nanofluid., 2011, 11, 623-631.

11 S. J. Kim, Y.-C. Wang, J. H. Lee, H. Jang and J. Han, Phys. Rev. Lett., 2007, 99, 044501.

12 C. C. Lai, C. J. Chang, Y. S. Huang, W. C. Chang, F. G. Tseng and Y. L. Chueh, Nano Energy, 2015, 12, 394-400.

13 Q. Pu, J. Yun, H. Temkin and S. Liu, Nano Lett., 2004, 4, 1099-1103.

14 P.-H. Chiu, C.-C. Chang and R.-J. Yang, Microfluid. Nanofluid., 2013, 14, 839-844.

15 S. Yu, T.-J. Jeon and S. M. Kim, Chem. Eng. J., 2012, 197, 289-294.

16 Z. Meng, Y. Chen, X. Li, Y. Xu and J. Zhai, ACS Appl. Mater. Interfaces, 2015, 7, 7709-7716.

17 C.-C. Chang, C.-P. Yeh and R.-J. Yang, Electrophoresis, 2012, 33, 758-764.

18 M. Taghipoor, A. Bertsch and P. Renaud, ACS Nano, 2015, 9, 4563-4571.

19 A. M. Benneker, J. A. Wood, P. A. Tsai and R. G. H. Lammertink, Sci. Rep., 2016, 6, 37236.

20 W. Guo, Y. Tian and L. Jiang, Acc. Chem. Res., 2013, 46, 2834-2846.

21 Y. Kong, X. Fan, M. Zhang, X. Hou, Z. Liu, J. Zhai and L. Jiang, ACS Appl. Mater. Interfaces, 2013, 5, 7931-7936.

22 Z. Zhang, X. Y. Kong, K. Xiao, Q. Liu, G. Xie, P. Li, J. Ma, Y. Tian, L. Wen and L. Jiang, J. Am. Chem. Soc., 2015, 137, 14765-14772.

23 X. Hou, Y. Liu, H. Dong, F. Yang, N. Li and L. Jiang, Adv. Mater., 2010, 22, 2440-2443.

24 X. Hou, H. Dong, D. Zhu and L. Jiang, Small, 2010, 6, 361-365.

25 M. Ali, B. Schiedt, R. Neumann and W. Ensinger, Macromol. Biosci., 2010, 10, 28-32.

26 Z. Guo, J. Wang, J. Ren and E. Wang, Nanoscale, 2011, 3, 3767-3773.

27 R. T. Perera, R. P. Johnson, M. A. Edwards and H. S. White, J. Phys. Chem. C, 2015, 119, 24299-24306.

28 L. Cao, W. Guo, Y. Wang and L. Jiang, Langmuir, 2012, 28, 2194-2199.

29 K. Xiao, P. Li, G. Xie, Z. Zhang, L. Wen and L. Jiang, RSC Adv., 2016, 6, 55064-55070.

30 W. J. Lan, M. A. Edwards, L. Luo, R. T. Perera, X. Wu, C. R. Martin and H. S. White, Acc. Chem. Res., 2016, 49, 2605-2613.
31 R. B. Schoch, J. Han and P. Renaud, Rev. Mod. Phys., 2008, 80, 839-883.

32 D. Woermann, Phys. Chem. Chem. Phys., 2003, 5, 1853-1858.

33 A. Mani, T. Zangle and J. Santiago, Langmuir, 2009, 25, 3898-3908.

34 F. H. J. Van Der Heyden, D. Stein and C. Dekker, Phys. Rev. Lett., 2005, 95, 9-12.

35 X. Jin, S. Joseph, E. N. Gatimu, P. W. Bohn and N. R. Aluru, Langmuir, 2007, 23, 13209-13222.

36 S. Movahed and D. Li, Electrophoresis, 2011, 32, 1259-1267.

37 T. Zangle, A. Mani and J. Santiago, Langmuir, 2009, 25, 3909-3916.

38 H. Hadidi and R. Kamali, J. Micromech. Microeng., 2016, 26, 035019.

39 A. Szymczyk, H. Zhu and B. Balannec, Langmuir, 2010, 26, 1214-1220.

40 M. Tagliazucchi and I. Szleifer, J. Phys. Chem. Lett., 2015, 6, 3534-3539.

41 S. Tseng, Y.-M. Li, C.-Y. Lin and J.-P. Hsu, Nanoscale, 2016, 2350-2357.

42 J. A. Wood, A. M. Benneker and R. G. Lammertink, J. Phys.: Condens. Matter, 2016, 28, 114002.

43 W. S. Koegler and C. F. Ivory, J. Chromatogr. A, 1996, 726, 229-236.

44 J. Wang, M. Zhang, J. Zhai and L. Jiang, Phys. Chem. Chem. Phys., 2014, 16, 23-32.

45 H. S. White and A. Bund, Langmuir, 2008, 24, 2212-2218.

46 C. Zhao, Y. Song and C. Yang, Phys. Fluids, 2015, 27, 012003.

47 Q. Liu, Y. Wang, W. Guo, H. Ji, J. Xue and Q. Ouyang, Phys. Rev. E: Stat., Nonlinear, Soft Matter Phys., 2007, 75, 051201.

48 C. Wei, A. J. Bard and S. W. Feldberg, Anal. Chem., 1997, 69, 4627-4633.

49 R. Qiao and N. R. Aluru, Appl. Phys. Lett., 2005, 86, 143105. 50 Y. Ai, M. Zhang, S. W. Joo, M. A. Cheney and S. Qian, J. Phys. Chem. C, 2010, 114, 3883-3890.

51 M. Tasaka, Pure Appl. Chem., 1986, 58, 1637-1646.

52 M. Tasaka, T. Mizuta and O. Sekiguchi, J. Membr. Sci., 1990, 54, 191-204.

53 W. B. Russel, D. A. Saville and W. R. Schowalter, Colloidal dispersions, Cambridge University Press, Cambridge, New York, 1989.

54 K. Kim, H. S. Kwak and T.-H. Song, Fluid Dyn. Res., 2011, 43, 041401.

55 G. Pardon and W. Van Der Wijngaart, Adv. Colloid Interface Sci., 2013, 199-200, 78-94.

56 R. Venditti, X. Xuan and D. Li, Microfluid. Nanofluid., 2006, 2, 493-499.

57 J. M. Xue, X. Q. Zou, Y. B. Xie and Y. G. Wang, J. Phys. D: Appl. Phys., 2009, 42, 105308.

58 F. M. Gilles, M. Tagliazucchi, O. Azzaroni and I. Szleifer, J. Phys. Chem. C, 2016, 120, 4789-4798.

59 S. S. Dukhin, Adv. Colloid Interface Sci., 1991, 35, 173-196. 60 M. Dietzel and S. Hardt, J. Fluid Mech., 2017, 813, 1060-1111. 